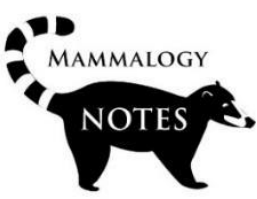

\title{
Primeros registros del género Caluromys (Didelphimorphia: Didelphidae) para el departamento del Quindío, y aclaraciones sobre algunos registros del occidente de Colombia
}

\author{
María Camila Bastidas-Domínguez 1* (D), Angélica Álzate Sánchez 2 (D), Sebastián O. \\ Montilla ${ }^{3-4}$ (D), Diana Carolina Valencia ${ }^{5}$ (D), Guillermo Cárdenas ${ }^{5}$ (iD), Hugo \\ Mantilla-Meluk 2-6 (iD)
}

1 Universidad de Nariño, San Juan de Pasto, Colombia.

2 Universidad del Quindío, Armenia, Colombia.

3 Fundación Proyecto Primates, Bogotá, Colombia.

4 Laboratorio de Ecología de Bosques Tropicales y Primatología - Universidad de Los Andes, Bogotá, Colombia.

5 Corporación Autónoma Regional del Quindío (CRQ).

6 Grupo CIDERA, Centro de Estudios de Alta Montaña - Universidad del Quindío, Armenia, Colombia.

*Correspondencia: k.dominguez238@gmail.com

\section{Resumen}

Se reportan los primeros registros del género Caluromys para el departamento del Quindío, en los Andes Centrales de Colombia. Estos registros corresponden a dos individuos de la especie C. derbianus, fotografiados en el municipio de Córdoba y uno más recuperado de una línea de alta tensión en el municipio de Pijao, al sur del departamento. De acuerdo con las nuevas localidades adicionadas en este estudio, además de registros de presencia contenidos en el repositorio GBIF y el análisis de registros en la literatura, se discute sobre la distribución de C. derbianus en Colombia y se hacen aclaraciones sobre los datos disponibles para esta especie en el occidente del pais. Finalmente, se presenta una herramienta visual para la identificación de las especies de la subfamilia Caluromyinae para Colombia.

Palabras clave: Andes, biodiversidad, C. derbianus, electrocución, Zarigüeya lanuda.

\begin{abstract}
We report the first records of the genus Caluromys for the department of Quindio, in the Central Andes of Colombia. These records correspond to two individuals of the species C. derbianus, photographed in the municipality of Córdoba and one individual recovered from a high voltage line in the municipality of Pijao, south of the department. According to the new localities added in this study, in addition to presence records contained in the GBIF repository and the analysis of records in the literature, the distribution of $C$. derbianus in Colombia is discussed and clarifications are made on the available data for this species in western of the country. Finally, a visual tool for the identification of the species of the Caluromyinae subfamily for Colombia is presented.
\end{abstract}

Key words: Andes, biodiversity, C. derbianus, electrocution, Woolly Possum 
La subfamila de marsupiales de América Tropical Caluromyinae (Kirsch 1977), distribuida en Centro y Sudamérica, incluye las zarigüeyas lanudas en los géneros Caluromys (Allen 1900), Caluromysiops (Sanborn, 1912) y Glironia (Thomas 1912). En ella, el género Caluromys, corresponde a animales arborícolas, nocturnos y solitarios de tamaño medio (140 a $400 \mathrm{~g}$ - 400 a 780 mm). Las especies de este género, se reconocen por las siguientes características: coloración dorsal rojiza con pelos lanosos largos (> $60 \mathrm{~mm}$ ), parches oscuros en los hombros, presencia de una línea facial oscura, orejas membranosas, hocicos puntiagudos con vibrisas desarrolladas, ojos prominentes, extremidades más claras (amarillentas, gris pálido al blanco), manos pentadáctilas y cola prensil tan larga como el cuerpo o más, usualmente cubierta de pelo hasta cierto porcentaje de su extensión (Duszynski 2016; Marineros et al. 2016).

a

b

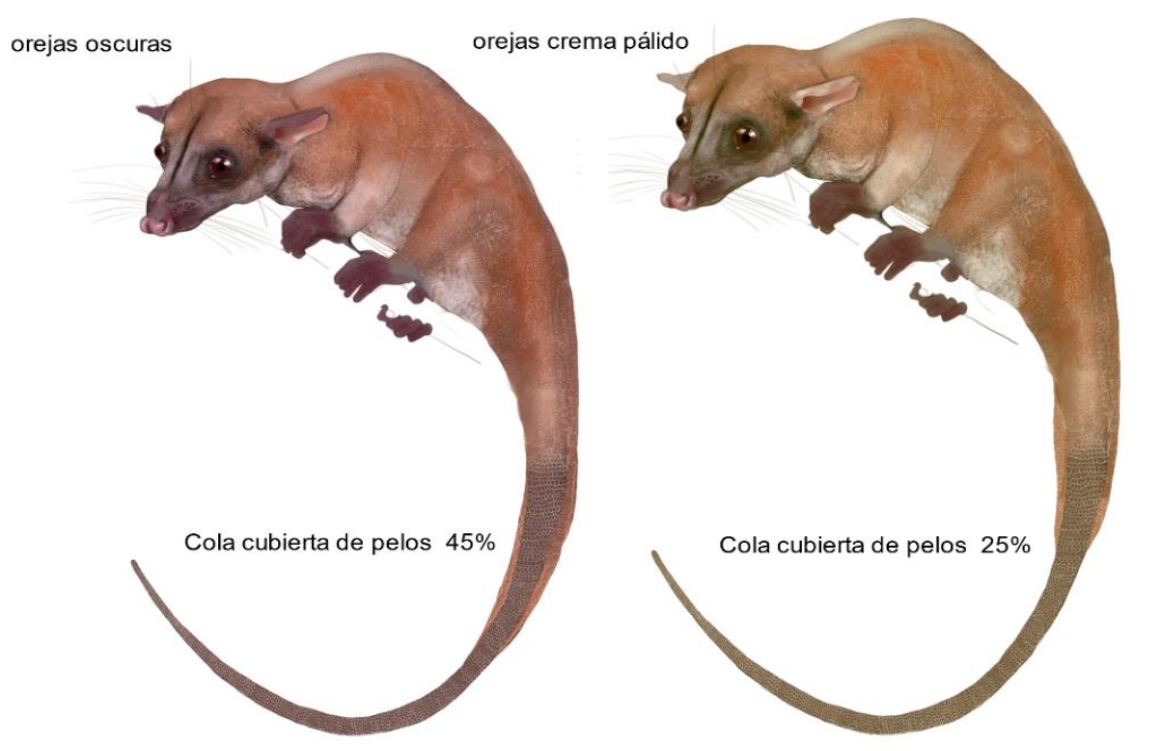

FIGURA 1. Caracteres externos que diferencian a las especies de chuchas lanudas: (a) Caluromys lanatus y (b) C. derbianus, presentes en el occidente de Colombia.

Se ha sugerido que las tres especies que conforman el género Caluromys, que es a su vez el de mayor extensión, se reemplazan geográficamente en distribución con algunas áreas de sobreposición, y además, pueden ser diferenciadas de acuerdo a caracteres externos de la siguiente forma: i) Caluromys derbianus Waterhouse 1841, con presencia desde el sur de México hasta el Noroccidente de América del Sur en Colombia y Ecuador (Bucher \& Hoffmann 1980; Gardner 2008; Voss \& Jansa 2009; Marineros et al. 2016); es la especie de mayor tamaño del género (promedio largo total $=414.5 \mathrm{~mm}$ ); tiene las orejas pálidas (blanco cremoso a rosado); y cobertura de pelo hasta aproximadamente la mitad de la cola. A pesar de la documentada variabilidad en color del pelaje corporal (Allen 1904), se ha sugerido que para $C$. derbianus, la coloración sobre los hombros y las extremidades es pálida, cuando se le compara con ii) C. lanatus Olfers 1818, de tamaño intermedio (promedio largo total $=382.5 \mathrm{~mm}$ ); orejas oscuras (café a negro); pelaje corporal rojizo, más oscuro sobre los hombros, y cola cubierta de pelo hasta tres cuartos de su extensión (Figura 1). Esta especie está distribuida desde la porción norte de la Sierra Nevada de Santa Marta, a lo largo de los Andes Orientales de Colombia y Venezuela; y los piedemontes andino-amazónicos de Ecuador y Bolivia, hasta Paraguay y el sur de Brasil (Allen 1904; 
Hershkovitz 1949; Gardner 2008). Finalmente, iii) C. phylander Linnaeus 1758, se asociada a ambientes de los escudos Guayanés (Venezuela, Guayana, Surinam y Guayana Francesa, y norte de Brasil) y Brasilia (sur de Brasil) y corresponde a la especie más pequeña del género (promedio largo total $=360 \mathrm{~mm}$ ), con orejas pálidas y cola cubierta de pelo entre un 10 y $20 \%$ de su extensión, con la porción desnuda café o moteada con marcas pálidas. Su pelaje dorsal es corto $(<65 \mathrm{~mm})$ y laxo, sin un patrón definido, excepto en su cara que es gris y que contrasta con las líneas faciales mediales y laterales, que son más oscuras, como sus anillos oculares (Emmons \& Feer 1999; Gardner 2008).

Para Colombia, se ha sugerido que $C$. derbianus se distribuye en ambientes de bosques entre los 200 y los 2.460 msnm, con registros para las regiones Andina y Pacífica en los departamentos de Antioquia, Caldas, Cauca, Chocó, Nariño, Risaralda, Tolima, y Valle del Cauca (Allen 1904; Sánchez \& Alvear 2003; Gardner 2008; López-Cepeda 2010; Solari et al. 2013; Cuartas-Calle \& Marín-Cardona 2014); y Caribe, con registros en el departamento de Córdoba (Gardner 2008); y Magdalena (Fonseca \& Astúa 2015). Recientemente, la especie ha sido reportada para el departamento de Caldas por Terán-Sánchez et al. (2021), de acuerdo con un avistamiento documentado en un proyecto de ciencia ciudadana; siendo a la fecha el departamento del Quindío, el único sobre la porción occidental de los Andes sin registros confirmados para esta especie. Cabe mencionar que la presencia del género Caluromys en el Quindío, ha sido sugerida a partir de encuestas a pobladores de veredas en el municipio de Génova (Parra-Colorado et al. 2014).

En este estudio, se reportan los primeros registros del género Caluromys para el departamento del Quindío, representados por material recuperado de un individuo de $C$. derbianus electrocutado al desplazarse por un cable de alta tensión en la zona periurbana del municipio de Pijao (Figura 2a-c) (4.3458", 75.7010", 1724 msnm). Además de dos individuos de la misma especie, registrados a partir de fotografias en el municipio de Córdoba (Figura 2d; Figura 2e).

El cuerpo del individuo de C. derbianus muerto por electrocución se recuperó el 31 de agosto de 2020, cuatro días después de su muerte, que se presume ocurrió el 27 de agosto del mismo año, día en que se observó al individuo electrocutado por primera vez. El ejemplar recuperado corresponde a un adulto de sexo indeterminado, el cual se encontraba en estado de descomposición y con quemaduras en la cabeza, porción ventral, los miembros anteriores y la cola (Figura 2a). Del ejemplar, se pudieron fotografiar partes de la anatomía y preservar parcialmente el cráneo y la mandíbula deteriorada. Al momento de la recuperación, el cuerpo en descomposición había sido colonizado por larvas de insectos (dípteros y coleópteros) y hormigas no determinadas. El individuo recuperado presentó orejas de color blanco cremoso, entre "pale salmon" a "sea shell pink"; con el dorso de color general rojizo pálido en los hombros variando del "ferrugineous" al "mars red" y una banda central clara, que vario del "salmon" al "apricot buff"; las extremidades anteriores y cola de color más claro, variando del "apricot buff" al blanco, según colores en Ridgeway (1912). Las medidas craneales documentadas siguieron a Voss \& Jansa (2009) y se resumen en la Tabla 1.

El ejemplar electrocutado fue asignado a C. derbianus de acuerdo con la descripción de la especie en la clave taxonómica para el género propuesta en Gardner (2008), así como comentarios en Allen (1904), que externamente lo asocian a las formas sudamericanas descritas por este autor, así como a material reportado por Hershkovitz (1949) del norte de Colombia. El cráneo del individuo junto a muestras de tejidos de músculo y pelo fue 
depositado en la Colección de Mamíferos de la Universidad del Quindío (CMUQ), material identificado con número de catálogo CMUQ 1280.

TABLA 1. Medidas del cráneo del individuo de Caluromys derbianus recuperado de un cable de alta tensión en Pijao. Quindío.

\begin{tabular}{ccc}
\hline Vista & Medida & $\mathrm{mm}$ \\
\hline Lateral & Altura del maxilar & 8.49 \\
& Longitud del orbital & 12.48 \\
Ventral & Longitud de la hilera dental superior & 28.71 \\
& Longitud del paladar & 62.82 \\
Dorsal & Longitud del nasal & 23.77 \\
& Ancho del zigomático & 36.91 \\
Lateral mandíbula & Longitud del coronoide & 9.11 \\
& Altura del dentario & 5.21 \\
& Longitud de la hilera dentaria & 20.6 \\
\hline
\end{tabular}

De otra parte, se tiene un primer registro fotográfico de un individuo vivo en el municipio de Córdoba, el cual fue logrado tras la evaluación del predio La Esmeralda (4.3844, -75.7152) (Figura 2d) por personal de la Corporación Autónoma Regional del Quindío (CRQ 2020a). El área del registro se asocia a un remanente de bosque que en la clasificación de zonas de vida de Holdridge (1967) se ubica como un bosque húmedo montano bajo y que según el mapa de ecosistemas terrestres del IDEAM (2011) corresponde a cobertura de bosque fragmentado con pastos y cultivos. Análisis previos de vegetación, adelantados por la CRQ (2020a), identificaron un total de 100 especies de plantas distribuidas en 58 familias, además de establecer al predio La Esmeralda como importante para la conservación en el municipio, púes en él se encuentra un nacimiento tributario de la Quebrada Sardineros. El remanente de bosque estudiado hace parte de un corredor biológico en el que se han documentado directamente otras especies de mamíferos, como Cerdocyon thous, Dasyprocta punctata, Dasypus novemcinctus y Notosciurus grantensis.

El registro fotográfico de $C$. derbianus corresponde a un animal de pelaje dorsal rojizo entre "vinaceous rufous" a "ferrigineous", con las orejas claras del blanco al rosado "pale brownish vinaceous"; las extremidades grisáceas "smoke gray" y cola blanca, y pelaje cubriendo hasta la mitad de la cola de color general blanco, colores según Ridgeway (1912). Al momento de ser fotografiado el animal se hallaba alimentándose en un árbol de mango (Mangifera sp.) (Figura 2d).

El segundo registro del municipio de Córdoba, corresponde a un individuo juvenil (Figura 2e) de la misma especie, publicado en un boletín de prensa el día 10 de septiembre de 2020 por la CRQ. Este individuo estaba en condiciones de cautiverio y fue entregado voluntariamente a la corporación después de la caída de una guadua que ocasionó la muerte a la madre. Las fotografias indican que el juvenil exhibía una coloración dorsal del "vinaceous rufous" al "raw siena", más pálida y menos rojiza (CRQ 2020b), al ser contrastadas con las fotografias del primer individuo fotografiado en el predio La Esmeralda. Las orejas de color rosa pálido "pale brownish vinaceous", la cara variando en tonos amarillentos "raw sienna" al gris "smoke gray" y la cola variando del café "Auburn" al blanco en su parte más distal, con un moteado café claro sobre las partes claras, según Ridgeway (1912), contrastando con la mayor extensión distal blanca de los adultos de Pijao y Córdoba.

Según nuestra revisión de registros en la literatura y bases de datos, resumimos que $C$. derbianus ha sido documentada para Colombia en las regiones Andina, Caribe y Pacífica, 
en los departamentos de: Antioquia, Caldas, Cauca, Cesar, Chocó, Córdoba, Magdalena, Nariño, y Valle del Cauca; sin embargo, para el departamento del Quindío no habían sido reportado hallazgos hasta la fecha, aunque eran esperados debido al patrón general de distribución que muestra esta especie en Colombia (Figura 3, Tabla 2).

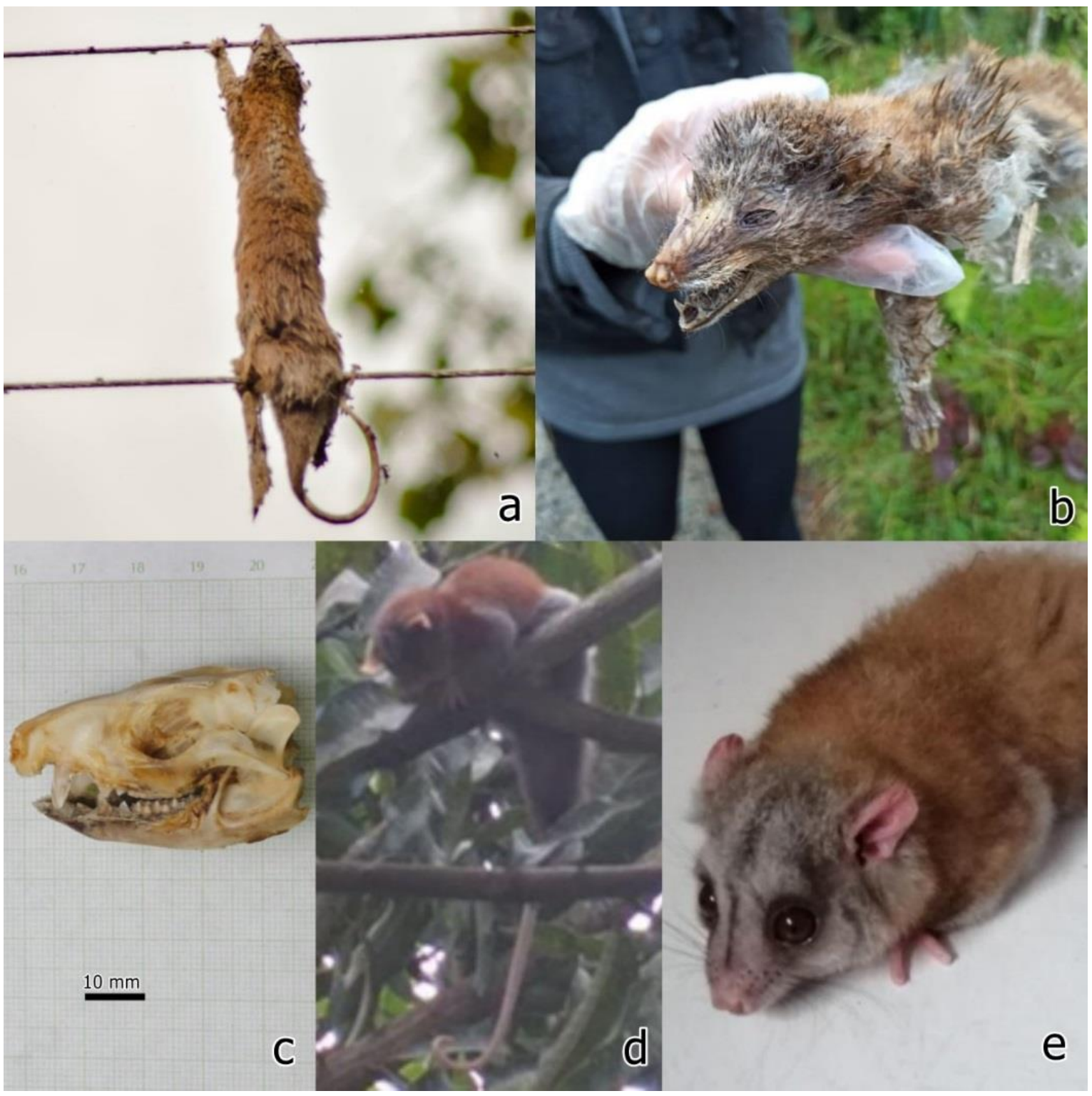

FIGURA 2. Individuos de C. derbianus del sur del Quindío. a. Hallazgo en el cableado eléctrico del área periurbana de Pijao, Quindío. b. Recuperación del ejemplar para su posterior preparación. c. Cráneo extraído. d. Individuo observado en el predio La Esperanza de Córdoba, Quindío. e. Individuo juvenil entregado voluntariamente por la comunidad del municipio de Córdoba a funcionarios de la CRQ.

En nuestro análisis sobre la procedencia del material colombiano reportado en la literatura, anotamos que en la obra de Gardner (2008) se hace referencia a material de soporte para el departamento del Valle del Cauca, identificado con un punto en inmediaciones de Buenaventura. Sin embargo, el texto de descripción del mapa menciona como localidad de muestreo para el departamento de Valle del Cauca el "Rio Caqueta" citando a Allen (1904) (Gardner 2008), quien hace referencia a material recolectado en 
Costa Rica por J. H. Batty: "None of these 1904 Allen, Mammals from Tropical America specimens agrees Caluromys laniger pallidus with what I take to be true derbianus, represented by four specimens from the Rio Cauqueta" (error de tipografia), "in the Cauca region of Colombia, which have the greater part of the dorsal aspect of the body, and also the limbs, intense light rufous, with a broad, very sharply defined gray shoulder-stripe."

A pesar de que en la base de datos del $A M N H$, el ejemplar en mención se identifica como un macho de C. derbianus derbianus (AMNH 15074), efectivamente recolectado por J. H. Batty, el 25 de junio de 1898, número de colector 484, en la localidad "Río Caquetá, departamento de Cauca", este individuo hace parte de la serie de ejemplares recolectados en "Río Cauquita" (Rio Cauqueta, error de escritura en el libro de accesión), correspondiente a un humedal en lo que hoy es el barrio Puerto Mallarino de Santiago de Cali, Valle del Cauca (Álvarez et al. 2009), que junto con los ejemplares: ơAMNH 14189-91, recolectados en agosto 1 de 1897; números de colector 564-66 de J. H. Batty, hicieron parte de los análisis de J. A. Allen. La comprensible confusión entre los departamentos Cauca y Valle del Cauca, en la base de datos del AMNH y reproducida en Gardner (2008), es debida a el error caligráfico de J. H. Batty sobre la localidad de muestreo en el libro de accesión y a los cambios en la división política de Colombia, ya que para 1904, año de la publicación de J. A. Allen, el departamento de Valle del Cauca hacía parte del departamento del Cauca, región a al que hace alusión el autor en su texto. El departamento de Valle del Cauca fue reconocido solo hasta 1910 (Decreto No 340 del 16 de abril, 1910).
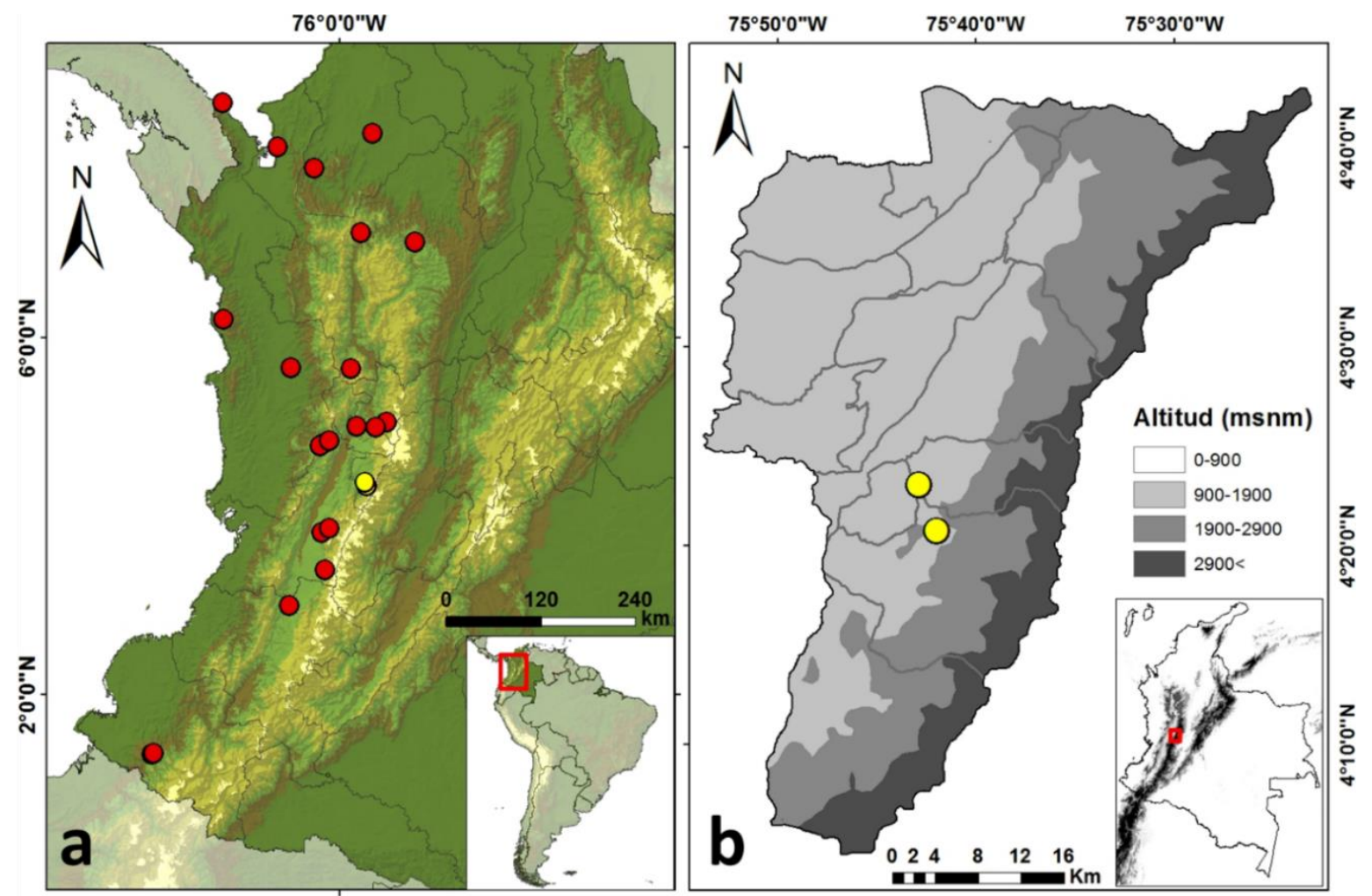

FIGURA 3. A., Registros de la especie C. derbianus en Colombia y B., nuevos registros en el departamento del Quindío. Los puntos rojos corresponden a la distribución histórica de la especie y los puntos amarillos a nuevos registros en el Quindío.

Esta aclaración cobra relevancia si se considera que en la descripción de C. derbianus por Waterhouse (1841) no se define una localidad tipo. Sin embargo, Cabrera (1958) y Gardner 
(2008), en sus recuentos de la taxonomía de la subespecie $C$. $d$. derbianus, mencionan que la localidad tipo para la subespecie nominal, fue asignada al Valle del Cauca, Colombia por Allen (1904).

En la publicación de Gardner (2008), notamos, que si bien el mapa de la Figura 3a, señala un punto en la región Caribe de Colombia, el mismo no se referencia en la descripción escrita de las localidades marginales para la especie. Debido a la escala del mapa, no es posible determinar si el punto hace referencia a una de las siguientes opciones: i) un espécimen macho de $C$. $d$. derbianus; recolectado por P. Hershkovitz; en abril 10 de 1949; y depositado en la Colección de Mamíferos del Field Museum of Natural History (FMNH 69327), proveniente del departamento de Córdoba, en el Rio Naín, en el alto Río Sinú; preservado como piel y cráneo, sin tejidos disponibles; o a ii) material depositado en el Museo Nacional de Historia Natural de los Estados Unidos, recolectado por Hershkovitz en Sierra Negra, Villanueva, Valledupar, el 3 y el 22 de diciembre (USNM 280903-280904) de 1942; y el 16 febrero de 1943 (USNM 280907). Este mismo material es citado por Fonseca \& Astua (2015) quienes lo refieren en los anexos de su revisión morfométrica del género, como proveniente del "Distrito de Magdalena". Es importante aclarar que Valledupar en la actualidad hace parte del departamento de Cesar, el cual se creó por la Ley 25 del 21 de junio de 1967. En nuestro conocimiento si existe material colombiano de C. derbianus proveniente del departamento de Magdalena, representado por un ejemplar macho recolectado por M. A. Carriker Jr., el 30 de diciembre de 1941 en la Hacienda Cincinnati, preservado como piel y cráneo (USNM 271317).

Hasta 1996, C. derbianus se consideraba como especie vulnerable (VU), pero en la última categorización para Colombia, la especie fue clasificada bajo la categoría de Preocupación Menor (LC, siglas en inglés) por la Unión Internacional para la Conservación de la Naturaleza (IUCN) (Lew et al. 2008). Pese al estatus asignado y según lo reportado por Castillo-Crespo \& González-Anaya (2007), algunas poblaciones de C. derbianus estarían decreciendo debido a la pérdida de hábitat por la expansión de los asentamientos humanos, situación particularmente cierta para los Andes Centrales de Colombia, donde existen altos índices de deforestación y transformación de las coberturas vegetales (González et al. 2011). El impacto de actividades antrópicas sobre la fauna andina, en particular sobre las especies de medianos y pequeños mamíferos constituye uno de los mayores vacíos de investigación. El establecimiento de asentamientos humanos ha incrementado la fragmentación de áreas boscosas, afectando principalmente la conectividad de hábitat propicios para especies arborícolas (Fonseca \& Astúa 2015; Montilla et al. 2020). Es en este sentido, los cableados eléctricos son cada vez más utilizados por especies de marsupiales, edentados, prociónidos y primates, ya que estos les permiten un rápido y fácil desplazamiento entes parches de bosque (Suárez-Cáceres et al. 2020). No obstantes, estas estructuras no están diseñadas para el paso de mamíferos y aparte del caso aquí registrado, se han encontrado mesomamíferos arborícolas muertos por electrocución para los Andes Centrales y Occidentales de Colombia incluyen: registros de $C$. derbianus en zonas rurales y periurbanas de Caldas y Valle del Cauca (Sánchez \& Alvear 2003; Rodríguez et al. 2018), un perro de monte Potos flavus en la ciudad de Armenia, depositado en la Colección de Mamíferos de la Universidad del Quindío; y diez individuos del mono nocturno andino (A. lemurinus) (Montilla et al. 2020). Por lo tanto, es necesario tomar medidas urgentes para mitigar esta problemática, específicamente en lugares donde constantemente estos mamíferos arboricolas utilizan estructuras antropogénicas para movilizarse, por lo que se sugiere aislar los cables de estas zonas con mayor tránsito 
o que se propongan alternativas como la construcción de estructuras que permita el paso sin mayor riesgo, como puentes ecológicos.

Si bien, el Quindío hizo parte de las expediciones más importantes de documentación de mamíferos adelantadas por el Museo Americano de Historia Natural a finales del XIX y principios del siglo XX en Colombia (Allen 1916), la historia de documentación para este grupo no ha tenido el mismo desarrollo experimentado por departamentos adyacentes (Caldas, Tolima y Valle del Cauca) con colecciones de mayor trayectoria en universidades de mayor tamaño. Es así como varias de las especies de mamíferos sugeridas para el departamento en listas nacionales, asumen la presencia de estos taxones debido a su documentación en departamentos vecinos (Alberico et al. 2000; Solari et al 2013). También, es de consideración señalar que en el Quindío, la mayor cantidad de investigaciones mastozoológicas se han realizado en la porción norte del departamento, en tanto la zona sur y suroriente cuenta con menos información sobre su fauna, situación principalmente asociada al histórico impacto del conflicto armado, con el páramo de Chili-Barragán el paso más bajo de conexión entre las vertientes oriental y occidental de la Cordillera Central, convirtiendo esta área en objetivo militar. Lo anterior ha dificultado el acceso a municipios como Pijao, Génova y Córdoba para el desarrollo de investigación científica (Gómez-Hoyos et al. 2014; Vásquez et al. 2019). Con la creación de la Colección de Mamífero del Quindío y sus proyectos académicos y de investigación asociados, ha sido posible un proceso sistemático de organización de la información mastozoológica, tendiente a la consolidación de una lista oficial de especies para el departamento. Avances previos en esta tarea los representan las documentaciones científicas con soporte museológico de Choloepus hoffmanni (Aguilar-Isaza \& Lopez-Obando 2009); Mustela frenata (Palacio et al. 2014), Dasyprocta punctata (Torres-Trujillo \& Mantilla-Meluk 2017), y Eptesicus chiriquinus (Gutiérrez-Cifuentes et al. 2013), especies consideradas comunes pero sin referencias departamentales, que han colateralmente permitido hacer aclaraciones importantes en la distribución nacional de estos taxones.

Los registros de $C$. derbianus presentados en este trabajo permiten complementar y esclarecer la distribución de la especie, la cual es ampliamente distribuida a lo largo de la Cordillera Occidental y parte de la Central de Colombia, asimismo, aporta al listado de mamíferos registrados para el Quindío, ya que en la zona sur y suroriente de la región presenta menor información sobre su fauna. Además, su presencia frecuente en zonas periurbanas, genera que sea imprescindible realizar estudios que se enfoquen en el conocimiento de la población en el departamento, identificando las rutas de mayor tránsito y promover estrategias para su conservación.

TABLA 2. Registros de la especie C. derbianus en las bases de datos de biodiversidad GBIF y SIB Colombia, museos y artículos científicos.

\begin{tabular}{|c|c|c|c|c|c|c|}
\hline Departamento & Municipio & Localidad & Latitud & Longitud & Altitud & Fuente \\
\hline Antioquia & Anorí & - & 7.07 & -72.15 & 1500 & GBIF \\
\hline Antioquia & Andes & - & 5.66 & -75.88 & 1400 & GBIF \\
\hline Antioquia & Ituango & - & 7.18 & -75.77 & 1500 & GBIF \\
\hline Antioquia & Turbo & - & 8.13 & -76.71 & - & GBIF \\
\hline Caldas & Manizales & $\begin{array}{l}\text { Vereda Hoyo } \\
\text { Frío }\end{array}$ & 4.99 & -75.59 & 1450 & MHNUCA:Mammal:383 \\
\hline Caldas & Manizales & $\begin{array}{c}\text { Vereda Hoyo } \\
\text { Frío }\end{array}$ & 4.99 & -75.59 & 1300 & MHNUCA:Mammal:248 \\
\hline Caldas & Belalcázar & Casco Urbano & 5.00 & -75.81 & 1550 & MHNUCA:Mammal:378 \\
\hline Caldas & Manizales & Río Blanco & 5.05 & -75.47 & 2560 & MHNUCA:Mammal:1801 \\
\hline
\end{tabular}




\begin{tabular}{|c|c|c|c|c|c|c|}
\hline Caldas & Manizales & $\begin{array}{l}\text { Vereda Hoyo } \\
\text { Frío }\end{array}$ & 4.99 & -75.59 & 1450 & MHNUCA:Mammal:382 \\
\hline Cauca & $\begin{array}{c}\text { Santander } \\
\text { de Quilichao }\end{array}$ & Mazamorrero & 3 & -76.58 & - & ROM 69493 \\
\hline Chocó & Unguia & - & 8.09 & -77.21 & - & FMNH 69801 \\
\hline Chocó & Unguia & - & 8.09 & -77.21 & - & FMNH 69802 \\
\hline Chocó & Unguia & - & 8.09 & -77.21 & - & FMNH 69803 \\
\hline Chocó & $\begin{array}{l}\text { Bahía } \\
\text { Solano }\end{array}$ & - & 6.21 & -77.33 & - & $\mathrm{GBIF}$ \\
\hline Chocó & Acandí & Capurganá & 8.63 & -77.34 & 25 & GBIF \\
\hline Chocó & Quibdó & Pacurita & 5.66 & -76.57 & 113 & $\mathrm{CMCH} 000020$ \\
\hline Córdoba & Planeta Rica & Marañonal & 8.29 & -75.63 & 72 & GBIF \\
\hline Córdoba & - & $\begin{array}{c}\text { Río Nain, Arriba } \\
\text { del Río Sinú }\end{array}$ & 7.9 & -76.3 & 200 & FMNH 69327 \\
\hline Nariño & Barbacoas & Junín & 1.33 & -78.15 & 800 & GBIF \\
\hline Nariño & Barbacoas & $\begin{array}{l}\text { Junín, La } \\
\text { Guarapería }\end{array}$ & 1.35 & -78.13 & 900 & GBIF \\
\hline Valle del cauca & El Cairo & $\begin{array}{l}\text { Corregimiento } \\
\text { Alto Bonito }\end{array}$ & 4.80 & -76.20 & 2120 & GBIF \\
\hline Valle del cauca & El Cairo & $\begin{array}{l}\text { Vereda } \\
\text { Vallecitos }\end{array}$ & 4.79 & -76.23 & 2395 & GBIF \\
\hline Valle del cauca & Pradera & $\begin{array}{c}\text { Corregimiento } \\
\text { San Isidro }\end{array}$ & 3.39 & -76.17 & 1960 & GBIF \\
\hline Valle del cauca & Guacarí & $\begin{array}{c}\text { Corregimiento } \\
\text { Santa Rosa de } \\
\text { Tapias }\end{array}$ & 3.82 & -76.21 & 2035 & GBIF \\
\hline Valle del cauca & $\begin{array}{c}\text { Guadalajara } \\
\text { de Buga }\end{array}$ & $\begin{array}{c}\text { Corregimiento } \\
\text { Crucero } \\
\text { Nogales, }\end{array}$ & 3.86 & -76.12 & 2550 & GBIF \\
\hline Valle del cauca & El Águila & $\begin{array}{c}\text { Corregimiento } \\
\text { Catarina-El } \\
\text { Embal }\end{array}$ & 4.84 & -76.13 & 2100 & GBIF \\
\hline
\end{tabular}

\section{AGRADECIMIENTOS}

A Daniel Pérez, por su apoyo en la recuperación del ejemplar electrocutado.

\section{REFERENCIAS}

Aguilar-Isaza N, López-Obando DC. 2009. Estado poblacional actual y hábitat del perezoso de dos dedos (Choloepus hoffmanni, Peters, 1858) en la reserva natural La Montaña del Ocaso, Quimbaya (Quindío), Colombia. Trabajo de grado, Carrera de Biología, Universidad del Quindío. Armenia, Colombia.

Allen JA. 1904. Mammals from southern Mexico and Central and South America. Bulletin of the American Museum of Natural History. 20:29-80. http:/ / hdl.handle.net/2246/1826

Allen JA. 1916. List of mammals collected in Colombia by the American Museum of Natural History expeditions, 1910-1915. Bulletin of the American Museum of Natural History. 35:191-238. http:// hdl.handle.net/2246/1826

Álvarez AL, Arana AE, Baena LM, Balanta C, Bolívar W, Calero A, Castro F, Flores PE, Gómez N, Gonzáles CA, et al. 2009. Los humedales del valle geográfico del Río Cauca, génesis, biodiversidad y conservación. Cali, Valle del Cauca: Corporación Autónoma Regional del Valle del Cauca.

Bucher J, Hoffmann R. 1980. Caluromys derbianus. Mammalian Species. 140:1-4. https://doi.org/10.2307/3504011/2600112

Cabrera A. 1958. Catálogo de los mamíferos de América del Sur. Revista del Museo Argentino de Ciencias Naturales ‘Bernardino Rivadavia. Ciencias Zoológicas 4:1-308. https:// doi.org/10.2307/1376929 
Castillo-Crespo LS, González-Anaya M. 2007. Avances en la implementación del Plan de Acción en Biodiversidad del Valle del Cauca. Cali, Valle del Cauca: Dirección Técnica Ambiental, Grupo Biodiversidad Corporación Autónoma Regional del Valle del Cauca.

Corporación Autónoma Regional del Quindío - CRQ. 2020a. Apoyo en la caracterización biológica en predios SIMAP; predio la esmeralda, SIMAP Córdoba, Quindío. Informe de gestión - Pág. 35.

Corporación Autónoma Regional del Quindío - CRQ. 2020b. Boletín de Prensa CRQ: Gestores de Córdoba recibieron una cría de zarigüeya lanuda. https://crq.gov.co/images/BoletinPrensa/2020/09.SEPTIEMBRE/BoletideprensaCRQ10deSeptiembre2020.pdf. Accessed on: 9-11-20.

Cuartas-Calle CA, Marín-Cardona D. 2014. Guía Ilustrada Mamíferos cañón del río Porce - Antioquia. Medellín, Colombia: EPM E.S.P, Universidad de Antioquia, Herbario Universidad de Antioquia.

Duszynski DW. 2016. Order Didelphimorphia-Eimeriidae. En: Duszynski DW, editor. The Biology and Identification of the Coccidia (Apicomplexa) of Marsupials of the World. Amsterdam, Países Bajos: Academic Press. p. 9-29. https://doi.org/10.1093/sysbio/syw006

Emmons LH, Feer F. 1999. Mamíferos de los bosques húmedos de América tropical: una guía de campo. Santa Cruz de la Sierra, Bolivia. Editorial F.A.N

Fonseca R, Astúa D. 2015. Geographic variation in Caluromys derbianus and Caluromys lanatus (Didelphimorphia: Didelphidae). Zoologia (Curitiba). 32(2):109-122. https://doi.org/10.1590/S198446702015000200002

Gardner AL. 2008. Mammals of South America. Chicago, Estados Unidos: The University of Chicago Press. p. 1127

Gómez-Hoyos DA, Ríos-Franco CA, Marín-Gómez OH, González-Maya JF. 2014. Representatividad de mamíferos amenazados en el sistema departamental de áreas protegidas (SIDAP) del Quindío, Colombia. Mammalogy Notes. 1(2):39-41. https:// doi.org/10.47603/manovol1n2.35-41

González JJ, Etter AA, Sarmiento AH, Orrego SA, Ramírez C, Cabrera E, Vargas D, Galindo G, García MC, Ordoñez MF. 2011. Análisis de tendencias y patrones espaciales de deforestación en Colombia. Bogotá, Colombia: Instituto de Hidrología, Meteorología y Estudios Ambientales-IDEAM.

Gutiérrez-Cifuentes DF, Brand-Alape A, Mantilla-Meluk H. 2013. Primer espécimen de la Colección de Mamíferos de la Universidad del Quindío (CMUQ): registro de Eptesicus chiriquinus (Chiroptera: Vespertillionidae) por atropellamiento en la Autopista del Café, Quindío, Colombia. Revista Biodiversidad Neotropical. 3(2):131-136.

Hershkovitz P. 1949. Generic names of the four-eyed pouch opossum and the woolly opossum (Didelphidae). Proceedings of the Biological Society of Washington. 62:11-12. https:// doi.org/10.2307/1382642

Holdridge LR. 1967. Life zone ecology. San José, Costa Rica: Tropical Science Center.

IDEAM. 2011. Análisis de tendencias y patrones espaciales de deforestación en Colombia. Bogotá, Colombia: Instituto de Hidrología, Meteorología y Estudios Ambientales-IDEAM.

Lew D, Soriano P, Cuarón AD, Emmons L, Reid F, Helgen K. 2008. Caluromys derbianus. The IUCN Red List of Threatened Species 2012: e.T3650A22175821. https://dx.doi.org/10.2305/IUCN.UK.20154.RLTS.T3650A22175821.en. Downloaded on 25 March 2021.

López-Cepeda NA. 2010. Evaluación preliminar de la distribución y abundancia relativa de mamíferos silvestres en el Santuario de Fauna y Flora de Otún Quimbaya mediante el uso de cámaras-trampa. Trabajo de grado Carrera de Biología, P. Universidad Javeriana.

Marineros LE, Vega HL, Adams J, McKewy M. 2016. Notas y nuevos sitios de encuentro de Caluromys derbianus (Marsupialia: Didelphidae) en Honduras. Revista Biodiversidad Neotropical. 6(1):77-84. https://doi.org/10.18636/ bioneotropical.v6i1.337

Montilla So, Rios-Soto JA, Mantilla-Castaño JC, Patiño-Siro D, Bustamante-Manrique S, Botero-Henao N, Ruiz S, Arias-Monsalve HF, Link A, Ramírez-Chaves HE. 2020. Eventos de electrocución de Aotus lemurinus (Primates: Aotidae) en los Andes Centrales de Colombia. Mammalogy Notes. 6(2):183. https://doi.org/10.47603/mano.v6n2.183

Mammalogy Notes 2021, 7(2), 218; https:// doi.org/10.47603/mano.v7n2.218

$10 / 10$ 
Palacio RD, Muñoz-Londoño AA, Mantilla-Meluk H. 2014. Primeros registros de la comadreja de cola larga Mustela frenata (Carnivora: Mustelidae) para el departamento del Quindío, Andes Centrales de Colombia. Biodiversidad Neotropical. 4 (2):170-176.

Parra-Colorado JW, Botero-Botero A, Saavedra-Rodrigez CA. 2014. Percepcion y uso de mamíferos silvestres por comunidades campesinas andinas de Genova, Quindío, Colombia. Bol. Cient. Mus. Hist. Nat. U. de Caldas, 18 (1): 78-93.

Ridgeway R. 1912.Colour standard and colour nomenclature. Baltimore, Estados Unidos: Hoen \& Co.

Rodríguez K, Lara HR, Ramírez S, Cedeño Y, Orrego CM, Pomarea E. 2018. Guía para la prevención y mitigación de la electrocución de la fauna silvestre por tendidos eléctricos en Costa Rica. San José, Costa Rica: Ministerio de Ambiente y Energía, Sistema Nacional de Áreas de Conservación.

Sánchez F, Alvear M. 2003. Comentarios sobre el uso de hábitat, dieta y conocimiento popular de los mamíferos en un bosque andino de Caldas, Colombia. Boletín Científico del Museo de Historia Natural Universidad de Caldas 7:121-144.

Solari S, Muñoz-Saba Y, Rodríguez-Mahecha JV, Defler TR, Ramírez-Chaves HE, Trujillo F. 2013. Riqueza, endemismo y conservación de los mamíferos de Colombia. Mastozoología Neotropical. 20(2):301-365.

Suárez-Cáceres GP, Adinolfi C, Sánchez F. 2020. Food selection and use of space by Didelphis pernigra (Didelphidae: Mammalia) in a suburban Andean environment. Acta Biológica Colombiana 25:368-373.

Terán-Sánchez S, Díaz-Arango A, Arias-Monsalve HF, Ramírez-Chaves HE. 2021. New records of mammals of the Coffee Region, Central Andes of Colombia using citizen science. Neotropical Biology and Conservation 16(1):27-43. https:// doi.org/10.3897/ neotropical.16.57932.

Torres-Trujillo N, Mantilla-Meluk H. 2017. Común e ignorado: ausencia de documentación científica del guatín Dasyprocta punctata (Rodentia: Dasyproctidae) en el departamento del Quindío, Colombia. Revista Biodiversidad Neotropical. 7(1):30-38. https:// doi.org/10.18636/ bioneotropical.v7i1.588

Vásquez P S, Chica-Galvis CA, Mantilla-Meluk H, Díaz-Giraldo V, Botero-Zuluaga M, Montilla S. 2019. Mamíferos en áreas de conservación de la Corporación Autónoma Regional del Quindío, Colombia. Biota colombiana, 20(2): 93-104. https:// doi.org/10.21068/c2019.v20n02a07

Voss R, Jansa S. 2009. Phylogenetic relationships and classification of didelphid marsupials, an extant radiation of New World metatherian mammals. Bulletin of the American Museum of Natural History. 322:1-177. https:// doi.org/10.1206/322.

Editor: Diego J. Lizcano

Received 2021-03-29

Revised 2021-04-15

Accepted 2021-05-18

Published 2021-07-07 